\section{Intraoperative neurophysiological monitoring improves safety in cervical chordomas surgery}

Gómez-Diago L. ${ }^{1}$, Hernández-Cádiz M.J. ${ }^{1}$, Torres-Caño N. ${ }^{2}$, Vicente-Fernández P. ${ }^{1}$, De Andrés J. ${ }^{1}$

${ }^{1}$ Consorcio Hospital General Universitario de Valencia., Dept of Anaesthesiology \& Intensive Care, Valencia, Spain, ${ }^{2}$ Hospital

Francisco de Borja, Neurophisiology, Gandia, Spain

\section{Background}

Chordomas are rare benign tumors that develop from embryonic notochord, can arise anywhere along the neuroaxis, being more unusual in the cervical spine. The treatment is based on surgery. The intraoperative neurophysiological monitoring (IONM) in cervical chordomas should include continous electroencephalogram (EEG) monitoring if a vertebral arteries compromise exists, as well as somatosensory (SEPs) and motor evoked potentials (MEP). Some anesthetic agents, like inhalational anesthetics and neuromuscular blocking drugs, might interfere in IONM.

\section{Case report}

A 69 yr-old woman with a history of cervical tumor, wich consists in a cervical C4-C6 chordoma lateralized to the right side. The patient refers right cervicobrachialgia and 2 months ago she presents an episode of respiratory insufficiency, needing an emergent tracheostomy. Due to the risk of the patient, a en bloc resection of the chordoma is scheduled in two surgical times. Previously to surgery, under radiological control a right vertebral artery occlusion test was done without any incidence in continuos neurological control. The IONM that is performed consists in the register of SEPs of lower limbs, MEP of upper and lower limbs and continuous EEG.

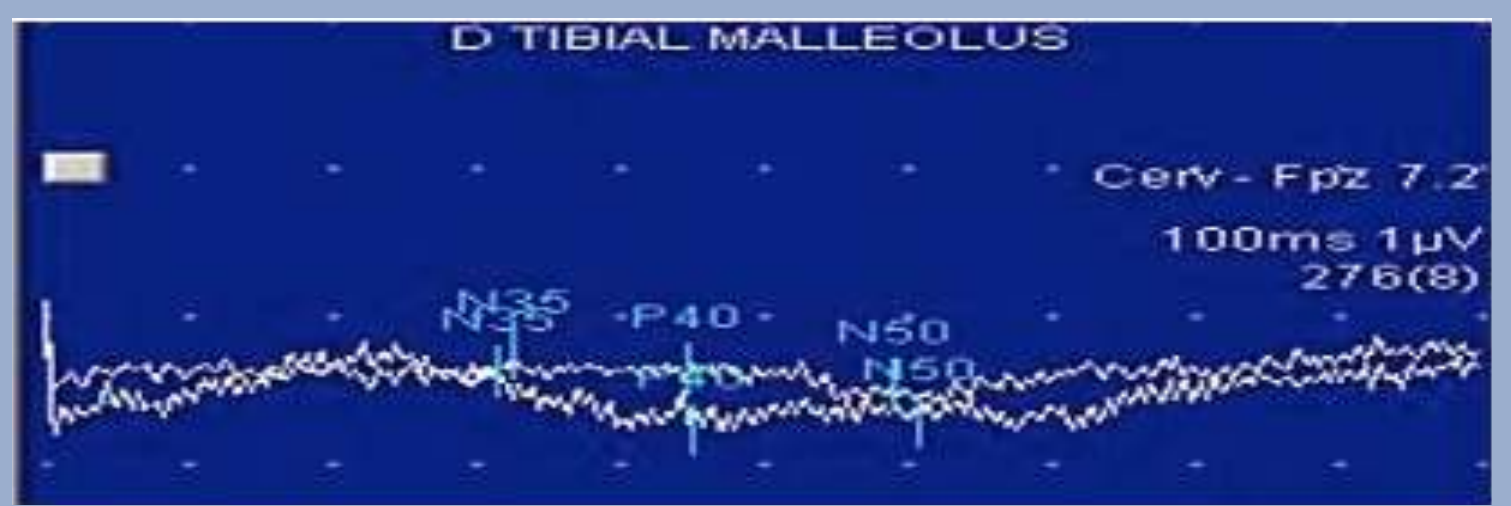

Image 1. Right SEPS loss

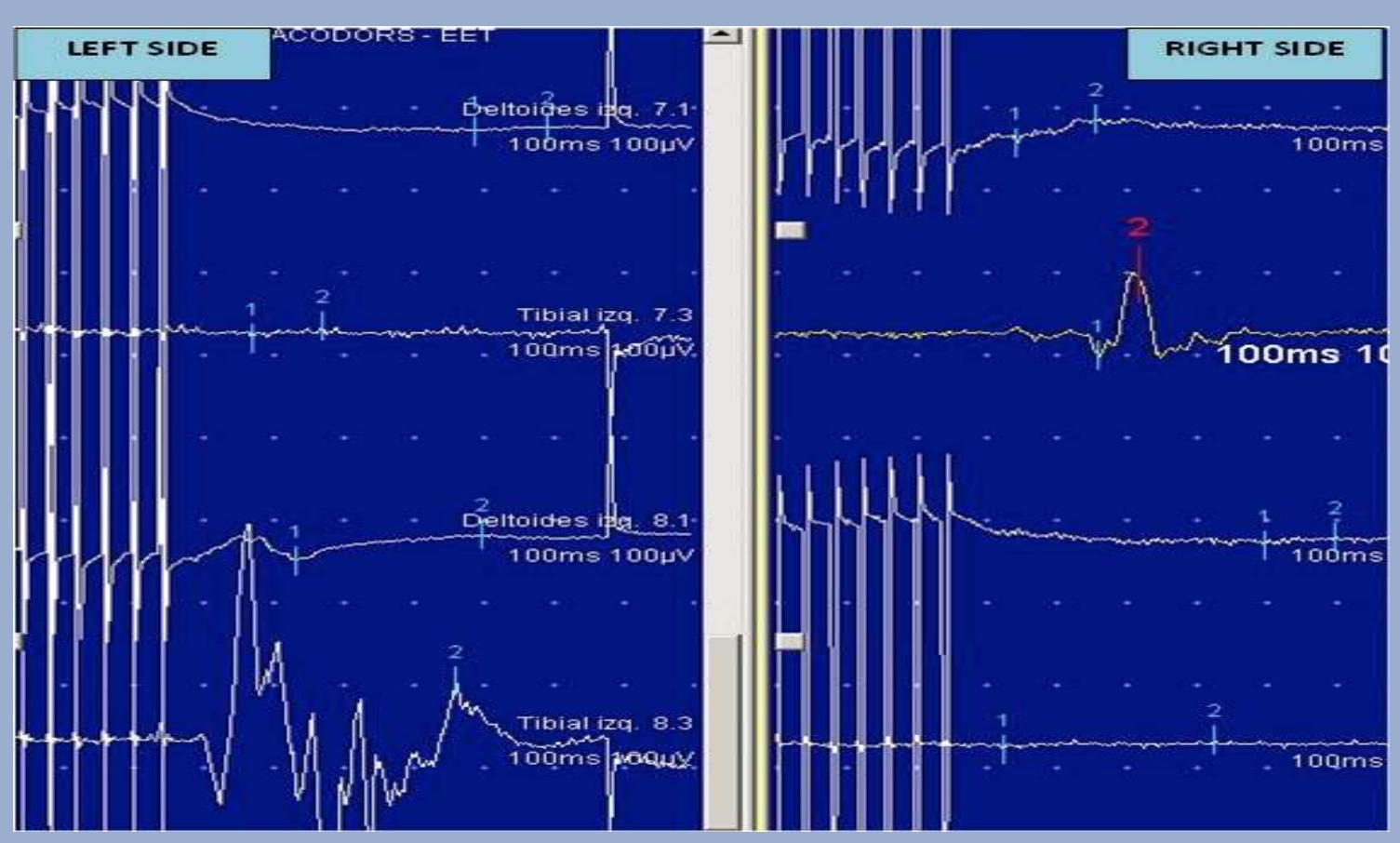

Image 2. MEP. Slight recovery of PEM in lower limbs, and very diminished responses in upper limbs (left side), being absent in right side.

\section{Discussion}

During the surgery, the ligature of the right vertebral artery was performed, observing a loss of right SEPS, which never recovered during the whole surgery. (Image 1)Moreover, a discrete reduction of the MEP was recorded (Image 2), without any specific valuable changes in the EEG, which is consistent with the test results of vertebral artery occlusion prior to surgery. During the IONM there was a close control of the use of anesthetic drugs to obtain the correct responses of the evoked potencials performed.

\section{Learning points}

The IONM through SEPs and MEP, as well as continuous EEG recording, are techniques which should be necessary in this kind of surgery. This set of techniques gives us more information at an early stage indicating the risk of neurological deficit and it also avoids complications during the anesthetic management. A close cooperation between the anesthesiologist and neurophysiologist is essential for the management of these patients. 